Article

\title{
An Online Super-Twisting Sliding Mode Anti-Slip Control Strategy
}

\author{
Zhiwu Huang ${ }^{1,2}$, Wei Du ${ }^{1,2}$, Bin Chen ${ }^{1,2}$, Kai Gao ${ }^{2,3}$, Yongjie Liu ${ }^{1,2}$ and Xuanheng Tang ${ }^{1,2}$ \\ and Yingze Yang $2,4, *$ (D) \\ 1 School of Automation, Central South University, Changsha 410114, China; hzw@csu.edu.cn (Z.H.); \\ 174611079@csu.edu.cn (W.D.); chenbinrxrg@163.com (C.B.); lyjajie@csu.edu.cn (Y.L.); \\ tangxuanheng@126.com (X.T.) \\ 2 Hunan Engineering Laboratory of Rail Vehicles Braking Technology, Changsha 410114, China \\ 3 College of Automotive and Mechanical Engineering, Changsha University of Science \& Technology, \\ Changsha 410114, China; kai_g@csust.edu.cn \\ 4 School of Computer Science and Engineering, Central South University, Changsha 410114, China \\ * Correspondence: Yangyingze@csu.edu.cn
}

Received: 13 February 2020; Accepted: 3 April 2020; Published: 9 April 2020

\begin{abstract}
The variability of rail surfaces can result in wheel-rail slippage, which reduces the accuracy of subway braking systems, or even endangers the operation safety. It is necessary to conduct optimal anti-slip control with the estimation of the wheel-rail adhesion state. In this paper, an online super-twisting sliding mode anti-slip control strategy is proposed for subway vehicles. Firstly, real-time wheel-rail adhesion state estimation is performed by utilizing the recursive least squares algorithm under complex and variable rail surface conditions. Then, the differential evolution algorithm is adopted to search the current optimal slip velocity based on the wheel-rail adhesion state. The super-twisting sliding mode controller is designed to implement the optimal sliding velocity tracking. The controller exploits the high-order derivatives of the sliding mode to eliminate chatter vibration and avoid the effect of disturbance, improving the anti-slip control performance. Finally, the effectiveness of the proposed anti-slip strategy is verified by experimental results.
\end{abstract}

Keywords: anti-slip control; recursive least rquare; differential evolution; super-twisting

\section{Introduction}

The subway braking system plays a vital role in the safe and reliable operation of a subway [1]. It is significant for a subway braking system to make the braking force tract the desired value. The braking of the subway relies on the friction between the brake shoes and the wheels of the basic braking part to reduce the speed of the subway wheels, and the deceleration of the subway car body depends on the adhesion of the rail surface. When the rail surface is slippery or the surface is oily, the adhesive force provided by the rail surface is not enough to support the required braking force. The wheels will slide on the rail surface, and the wheel tread and the rail surface will be heated sharply [2]. In this way, the braking performance will be lost, the braking distance will be longer, and the ride comfort will be reduced. At the same time, it will bring additional impact to the parts of the subway vehicle, accelerate its wear, and even endanger driving safety. In order to effectively use the adhesion between the wheels and rails and prevent the wheels from slipping, an anti-slip control system in the subway braking system is essential.

As one of the key subsystems to ensure driving safety, the anti-slip control system of subway vehicles has attracted increasing attention. During train operation, the adhesion force decreases as the slip velocity increases $[3,4]$. The anti-slip control of trains is mainly realized indirectly by controlling 
the slip velocity [5]. The control methods based on slip velocity mainly include (i) tracking of ideal slip velocity and (ii) obtaining the maximum adhesion force [6,7]. An anti-slip control method based on fuzzy logic was proposed by Nabizadeh [8] and Park [9]. This method divides the train slip velocity into different sections, and specifies different control strategies for the slip speed in different sections. A method using language and number system information was proposed by Precup et al. [10]. A predictive control method was proposed by Sajad Sad et al. [11] to predict the maximum adhesion coefficient that a train can obtain, which is expected to keep the train at this maximum adhesion coefficient. A new estimation method used higher-order sliding mode observer was proposed by [12] which aimed to address the problem of the road adhesion coefficient in the vehicle dynamics being unknown and varying with the contact surface. A simple but effective algorithm to estimate the slip ratio without using any information of vehicle velocity and dynamic parameters, which are very difficult to be determined, was proposed by [13]. However, the estimation period was a little long and did not suit subways. the above method is insufficient because the optimal slip velocity to be tracked is set offline, and the slip velocity of the train is controlled on the pre-set curve during operation. Besides, the complex wheel-rail contact conditions and the changing load of the subway make the adhesion force different under the same track surface conditions.

In recent years, many data-driven methods have been carried out to reduce the model dependence of anti-slip control and accurately estimate the adhesion state. The likelihood estimation was utilized in [14] to estimate the current wheel-rail adhesion state of the train. Wheel-rail adhesion parameters are obtained using the maximum likelihood estimation from the speed and other data collected by the train sensors. In $[15,16]$, the adhesion coefficient was detected as a function of slip velocity. The measured wheel velocity was used as input to the extended kalman filter to estimate the slip velocity. The authors in [17] presented real-time estimation of wheel-rail contact forces and moments based on an extended kalman filter estimator in normal driving conditions. A multiple model approach based on swarm intelligence to estimate friction at the wheel-rail interface was proposed in [18]. However, these methods suffer from limitations, such as the estimation speed being slow, and requiring more data samples at one time to obtain a more accurate estimation value. Furthermore, the limited computing resources of the industrial personal computer in a subway can not estimate the adhesion state in a short time.

To summarize, the current anti-slip control strategies by setting the optimal slip velocity are not suitable for the conditions of complicated and changing rail surfaces. Although the LuGre friction model used by $[19,20]$ can appropriately reduce the errors caused by complex rail conditions, the complexity of the model will cause the controller operation cycle to be longer. A new adaptive sliding mode control (ASMC) method based on pole-placement control (PPE) and a time-delay estimation (TDE) algorithm was proposed by [21,22], which can achieve fast fast adaptation and powerful abilities for tracking and chattering reduction. Because the structure of the control method is more complex, the calculation amount is higher, and the calculation capacity of the on-board controller of the subway train is limited, the method is not suitable for anti-slip control. Date-driven methods benefit from free model and real-time adhesion state. However, a large amount of data and strong computing power are required to support parameter estimation, which are unrealistic inside a subway.

To address the above problems, a new online anti-slip control strategy is proposed in this paper. Firstly, the recursive least squares is used to estimate the adhesion state of the subway wheel-rail online. The time-varying parameters in the adhesion model are continuously estimated by the recursive least square method using the sensor data in real time. In this way, a more accurate wheel-rail adhesion state could be obtained in a very short time with limited sensor data. Due to the limited computing capacity of the anti-slip chip inside the subway, direct differential calculation is very difficult. The differential evolution algorithm is adopted to search the current optimal slip velocity corresponding to the maximum value in the current non-linear wheel-rail adhesion curve. It avoids computing differentials and falling into local optimums compared with traditional optimization methods such as 
Newton's method. The super-twisting sliding mode controller is designed to implement the optimal sliding velocity control target. The chatter vibration could be eliminated and the anti-slip control performance could be improved using the designed controller compared with a traditional sliding mode control.

The main contributions of this paper are as follows:

- Recursive least squares is used to estimate the adhesion state between wheels and rails online, and the differential evolution algorithm is used to search the current optimal slip velocity. The online estimation method proposed in this paper can estimate the current wheel-rail state and search for the optimal slip velocity in 400 milliseconds, ensuring the real-time performance of subway brake control.

- the chattering of the sliding mode controller in the steady state is analyzed theoretically in this paper, and a super-twisting sliding mode controller is designed to eliminate the chattering.

- the proposed anti-slip strategy is verified by comparing with the traditional sliding mode control. The experimental results show that the method proposed in this paper can significantly improve the performance of control and eliminate chattering.

The remainder of the paper is organized as follows: In Section 2, the wheel-rail contact model and the subway single wheel pair kinematics model are established, and the adhesion characteristic curve is described and analyzed. Section 3 presents methods for online estimation of the adhesion state of subway wheels and rails and searching for optimal slip velocity based on the current adhesion state. In Section 4, a super-twisting sliding mode controller is designed to reduce the control errors and eliminate chattering. In Section 5, the effectiveness of the proposed method is validated through experiments. Section 6 draws the conclusion.

\section{Problem Formula and System Modeling}

\subsection{Wheel-Rail Contact Model}

The force analysis of the wheelset is shown in Figure 1. $\mathrm{mg}$ is vehicle axle weight, $f$ is air resistance, $T_{b}$ is braking torque. $F_{a}$ is adhesion force.

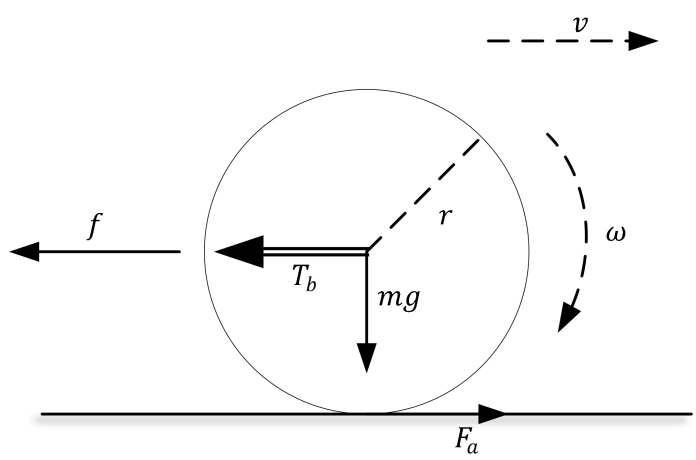

Figure 1. Wheel-rail contact force analysis.

During the braking process, due to the application of braking torque, the contact point of the wheel to the rail tends to slide to the right, and the load of the wheel causes the wheel to be tightly pressed onto the rail surface, forming a slight deformation. This deformation causes a small amount of sliding between the contact surfaces of the wheel and rail. This small amount of sliding is called creeping. Since only the tangential force can be transmitted by the static friction force generated by creep, the creep phenomenon is a normal phenomenon when the wheel is rolling. 
The occurrence of creep will cause the vehicle body linear speed $v$ not to be equal to the wheel linear speed converted by the wheel speed $v_{\omega}$. And during braking, $v$ will always greater than $v_{\omega}$. Use slip velocity $v_{s}$ to define the difference between the two speeds.

$$
v_{s}=v-r \omega
$$

where $r$ is wheel radius, $\omega$ is wheel angular velocity, $v$ is vehicle body linear velocity. In additional, the ratio of the slip velocity to the linear velocity of the car body is defined as the slip ratio, and its mathematical expression is

$$
\eta=\frac{v_{s}}{v}
$$

The adhesion coefficient is defined as the ratio between the tangential force and the normal load between the wheel and the rail, and its mathematical expression is

$$
\mu=\frac{F_{a}}{m g}
$$

\subsection{Adhesive Characteristic Curve Analysis}

The adhesion coefficient $\mu$ is determined by the physical contact state between the wheels and rails. This coefficient can intuitively reflect the adhesion state between the wheels and rails. Many factors are affected by the adhesion coefficient, therefore, in the actual operation of subway vehicles, the adhesion coefficient is a time-varying state quantity. The relationship between the adhesion coefficient and the slip velocity can be expressed as follows [23].

$$
\mu\left(v_{s}\right)=c_{1} e^{-a v_{s}}-c_{2} e^{-b v_{s}}
$$

where $c_{1}, c_{2}, a, b$ are parameters related to the physical conditions of wheel and rail contact. According to [24], we can know the experience value under the dry rail surface, wet rail surface and snow or ice rail surface as shown in Table 1.

Table 1. Parameters of Burckhardt's mode.

\begin{tabular}{ccccc}
\hline Rail Surface Conditions & $\boldsymbol{c}_{\mathbf{1}}$ & $\boldsymbol{c}_{\mathbf{2}}$ & $\boldsymbol{a}$ & $\boldsymbol{b}$ \\
\hline Dry & 1.0 & 1.0 & 0.54 & 1.2 \\
Wet & 0.29 & 0.29 & 0.54 & 1.2 \\
Ice & 0.1 & 0.1 & 0.54 & 1.2 \\
\hline
\end{tabular}

As can be seen from the Table 1, under different rail surface contact conditions, the values of parameters $c_{1}, c_{2}, a, b$ in Equation (4) are different, and there is a large difference between the three.

Although there are large differences in the above-mentioned three kinds of rail surface adhesion characteristic curves, the overall change trend of the three curves is the same. It can be seen from Figure 2 that the entire curve includes two parts; the adhesion area and the sliding area, and the adhesion area contains two parts, the linear area and the non-linear area. When the slip velocity is less than a certain value, the adhesion coefficient increases linearly with the increase of the slip velocity, and then the adhesion force gradually increases slowly. When the slip velocity reaches the highest point, the adhesion force also reaches the maximum value. At this time, if the braking force is increased, the slip velocity will be further increased and the slip zone will be affected, which will seriously affect the braking performance. It hurts the tread and rails of the wheels, and even endangers driving safety. 


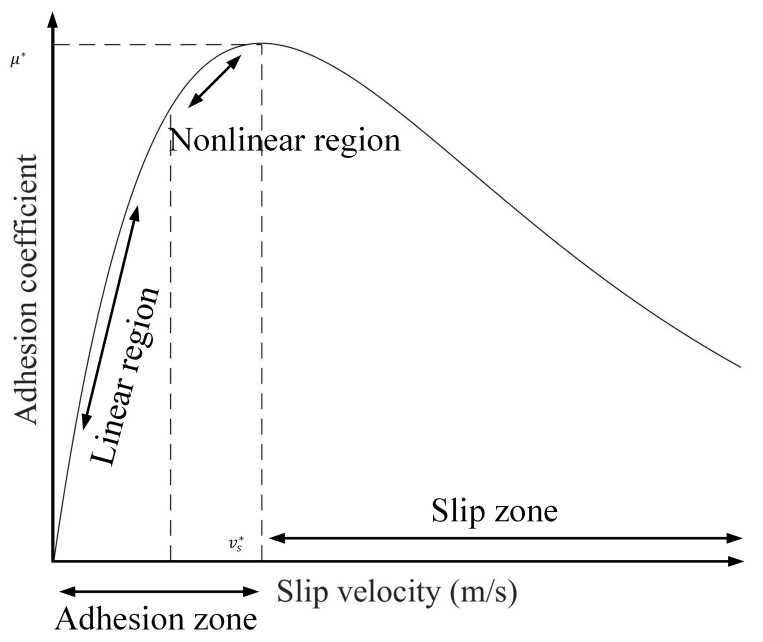

Figure 2. Detailed description of slip speed-adhesion curve.

In addition, due to the characteristics of tight departures and large changes in passenger capacity, the subway should actively estimate the current wheel-rail adhesion state of the train in real time during operation, calculate the braking force based on the currently required deceleration, and determine from the adhesion state curve meets the required braking force. If not, the output of the braking torque should be controlled so that the subway slip velocity is stable near the optimal slip velocity point.

\subsection{Kinematics Model of Subway Single Wheel Pair}

The force analysis diagram of the subway during operation is shown in the Figure 3.

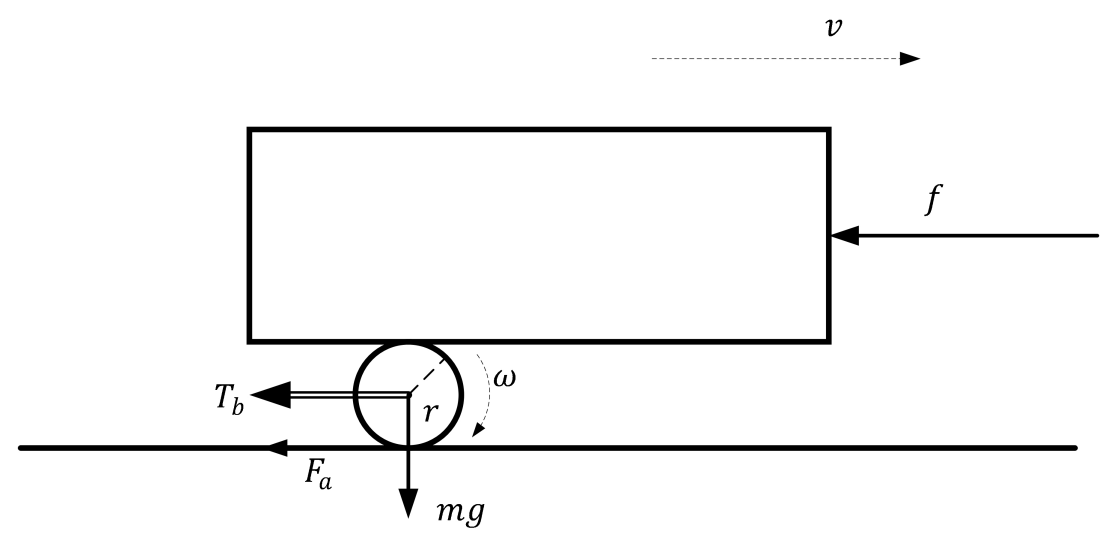

Figure 3. The force analysis diagram of the subway.

And its dynamics modeling can be described by the following mathematical form

$$
\begin{gathered}
m \dot{v}+F_{a}+f+W=0 ; \\
J \dot{\omega}+T_{b}+F_{a} r=0 \\
f=\mu_{0}+\mu_{1} v+\mu_{2} v^{2} \\
F_{a}=\mu\left(v_{s}\right) m g \\
v_{s}=v-\omega r
\end{gathered}
$$




$$
W= \begin{cases}\frac{600}{R} & , l_{r}<l_{t} \\ \frac{600}{R} \cdot \frac{l_{r}}{l_{t}} & , l_{r} \geq l_{t}\end{cases}
$$

where $m$ is wheel load, $F_{a}$ is the adhesion force of the track surface to the wheel, $g$ is gravitational acceleration, $f$ is air resistance, $\mu_{0}, \mu_{1}, \mu_{2}$ are resistance coefficients which are constant value in this paper, $v$ is vehicle body linear velocity, $J$ is wheel inertia, $r$ is wheel radius, $\omega$ is wheel angular velocity and $T_{b}$ is braking torque. Besides, $W$ is the additional resistance of the subway curve is related to the length of the curve. $l_{t}$ is the length of subway and $l_{r}$ is the arc length of the curve. $R$ is the curvilinear radius of the rail.

Because of the highly time-varying and non-linear characteristics of the changes in the load of a single wheel during the operation of a subway vehicle, and the intensive operation of subway vehicles, the accuracy of the parking requirements is extremely high, so the braking force control requirements are very precise. The braking force needs to be transmitted by the adhesion force between the wheels and rails. Therefore, the adhesion coefficient curve corresponding to the slip velocity of the subway vehicle needs to be estimated online in real time in order to select the optimal slip velocity for tracking control during braking. The architecture of the online super-twisting sliding mode anti-slip control strategy is shown in Figure 4.

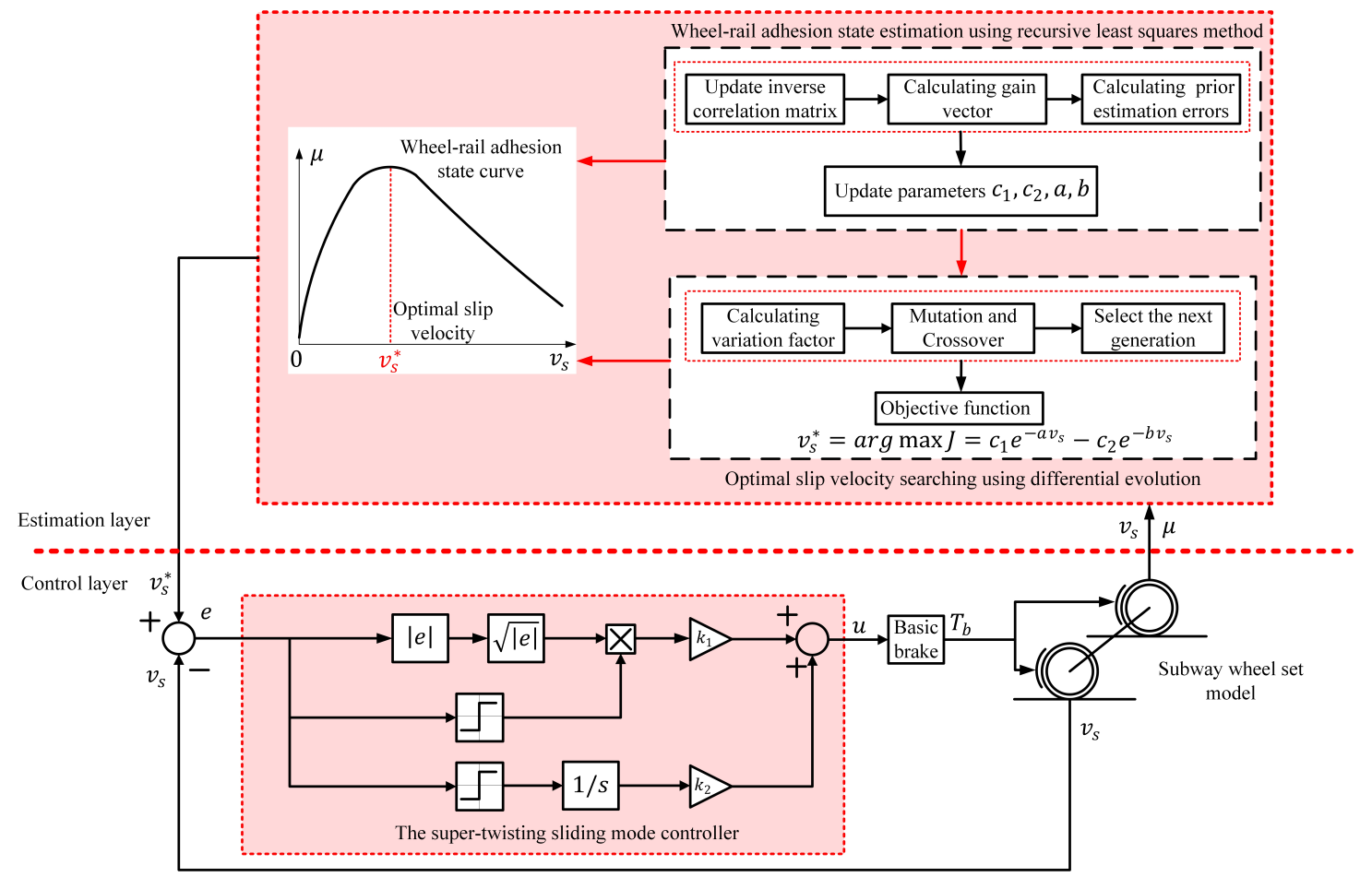

Figure 4. The architecture of the online super-twisting sliding mode anti-slip control strategy.

\section{Online Estimation of Optimal Slip Velocity}

\subsection{Estimating Wheel-rail Adhesion Status Online}

Due to the high real-time requirements of subways, online estimation based on complex algorithms such as machine learning is no longer used in this scenario. This paper selects the recursive least squares method for online parameter estimation, which can guarantee the high real-time requirements of subways.

In the light of the subway train dynamic model established in Equations (5)-(9), the train can obtain the vehicle body linear velocity and wheel angular velocity through sensors, and obtain the current 
instantaneous adhesion coefficient and current slip velocity through calculation. According to the internal data transmission protocol of subway, speed data. The sampling period is $16 \mathrm{~ms}$.

According to the mathematical model of slip velocity and adhesion coefficient used in Section 2, the function to be estimated is

$$
\mu=c_{1} e^{-a v_{s}}-c_{2} e^{-b v_{s}}
$$

where $c_{1}, c_{2}, a, b$ are parameters to be estimated. Let $\beta=\left[c_{1}, c_{2}, a, b\right]^{T}$ and $\hat{v}_{s_{i}}, \hat{\mu}_{i}$ are the values of actual measurement. Since the estimates are conducted online, $\hat{v}_{s_{i}}, \hat{\mu}_{i}$ is constantly changing. Therefore, the optimal parameter vector $\beta$ needs to be updated in each estimation cycle. So the recursive least squares method is choosen.

The estimated error for each data sample is

$$
\varepsilon_{i}=\hat{\mu}_{i}-\left(c_{1} e^{-a \hat{v}_{s_{i}}}-c_{2} e^{-b \hat{v}_{s_{i}}}\right)
$$

which can be writen as

$$
\varepsilon_{i}=\hat{\mu}_{i}-\mu\left(\beta_{i}, \hat{v}_{s_{i}}\right)
$$

And the optimal objective function is

$$
\phi\left(\beta_{n}\right)=\sum_{i=1}^{n} \lambda^{n-i} \varepsilon_{i}^{2}+\sigma \lambda^{n}\left\|\beta_{n}\right\|^{2}
$$

where $n$ is number of samples. The first term of the above formula is the weighted sum of squared residuals, $\lambda^{n-i}$ is an exponentially decaying weighting factor, also called forgetting factor. The introduction of the forgetting factor is to ensure that the long-term past data is "forgotten", and the latest data is tracked in time, and $0<\lambda<\approx 1$. The ordinary least squares method assumes that the weight of the measurement data is the same at each moment, which is unreasonable in the application scenario of this paper. If a measurement value has passed for a long time, its impact on the calculation result should be weakened, and the impact of newest data should strengthen. The second term of the above formula is the added regularization term to facilitate the derivation. Over time, $\lambda^{n}$ will tend to 0 , and the impact of regularization will become smaller and smaller. Besides, $\sigma$ is regularization coefficient, which is $\sigma>0, \sigma \in \mathbb{Z}$.

The calculation steps are as follows: initialization $\beta_{0}=\mathbf{0}, P_{0}=\sigma^{-1} I$, for every moment $k$,

Update inverse correlation matrix $P_{k}$ :

$$
P_{k}=\lambda^{-1} P_{k-1}-\lambda^{-1} g_{k} \hat{v}_{s_{k}}^{T} P_{k-1}
$$

where $\mathbf{g}_{k}$ is gain vector. Here's how to calculate. The intermediate quantities $\pi_{k}$ can be obtained by

$$
\pi_{k}=P_{k-1} \hat{v}_{s_{k}}
$$

The gain vector $g_{k}$ can obtained by

$$
g_{k}=\frac{\pi_{k}}{\lambda+\hat{v}_{s_{k}}^{T} \pi_{k}}
$$

Then the prior estimation errors $\xi_{k}$ can be obtained by

$$
\xi_{k}= \begin{cases}\hat{\mu}_{k}-\mu\left(\beta_{k-1}, \hat{v}_{s_{k}}\right) & \hat{\mu}_{k}-\mu\left(\beta_{k-1}, \hat{v}_{s_{k}}\right)>0 \\ 0 & \hat{\mu}_{k}-\mu\left(\beta_{k-1}, \hat{v}_{s_{k}}\right) \leq 0\end{cases}
$$


Finally update parameters $\beta_{k}$ according to the prior estimation errors

$$
\beta_{k}=\beta_{k-1}+g_{k} \xi_{k}
$$

\subsection{Search for Optimal Slip Speed Using Differential Evolution}

Through the recursive least squares method, the parameters $c_{1}, c_{2}, a, b$ value in Equation (4) can be estimated online, and the current wheel-rail adhesion curve can be obtained. In the subway braking, in order to maximize the use of adhesion, the slip velocity should be controlled near the optimal slip velocity. It can be seen from the adhesion characteristic curve that there is an optimal slip velocity regardless of the wheel-rail contact conditions. Because the curve is a non-linear function, obtaining extreme points directly through the derivation method will make the calculation time too long and there will be calculation errors, which will worsen the real-time performance of the online estimation. On the other hand, the computing power of the subway is very limited, especially it does not support direct differentiation operation. Therefore, this paper uses the differential evolution algorithm to obtain the best slip velocity. It avoids computing differentials and falling into local optimums compared with other optimization methods such as Newton's method. Although it is also an iterative algorithm, this algorithm does not need to perform a lot of matrix calculations, so the calculation speed will be much faster for subways. Its objective function is

$$
J\left(v_{s}\right)=\max _{0<v_{s} \leq 10} c_{1} e^{-a v_{s}}-c_{2} e^{-b v_{s}}
$$

where $c_{1}, c_{2}, a, b$ are parameter values estimated in real time for the previous step. When the objective function takes the maximum value, the value of $v_{S}$ is the current best slip velocity.

$$
v_{s}^{*}=\arg \max _{v_{s}} J\left(v_{s}\right)
$$

The differential evolution algorithm is used to obtain the maximum value of the above objective function, and the initial value is set to $v_{s}(0)=0.01$. The size of the mutation factor determines the diversity of the population. When the orbital surface environment does not change rapidly, the evolution equation will not change too much, and the change factor should be small. In addition, by accelerating the convergence of the algorithm, unnecessary calculations can be avoided. When the evolution equation changes significantly, the change factor should be larger to ensure that the algorithm jumps faster from the local extremes. With the help of logic functions, the mutation factor is designed as follows

$$
F(k)=F_{0} \cdot 2^{e^{1-\frac{G_{m}}{G_{m}+1-G(k)}}}
$$

where $G_{m}$ is the maximum number of iterations, $G(k)$ is current iterations and $F_{0}$ is base variation factor. The parameter settings of the entire algorithm are shown in the following Table 2.

Table 2. Parameters of differential evolution algorithm.

\begin{tabular}{cc}
\hline Parameters & Values \\
\hline Population size & 50 \\
Number of parameters & 1 \\
Variance factor & $F(k)$ \\
Cross factor & 0.6 \\
Max number of iterations & 300 \\
Parameter search range & $v_{s} \in[0.01,10]$ \\
\hline
\end{tabular}


Algorithm 1 describes the flow of the algorithm. First, the parameters of the differential evolution algorithm are initialized in accordance with Table 2. Then proceeds in the loop to find the extremes. Finally, the online estimation of the parameter is achieved.

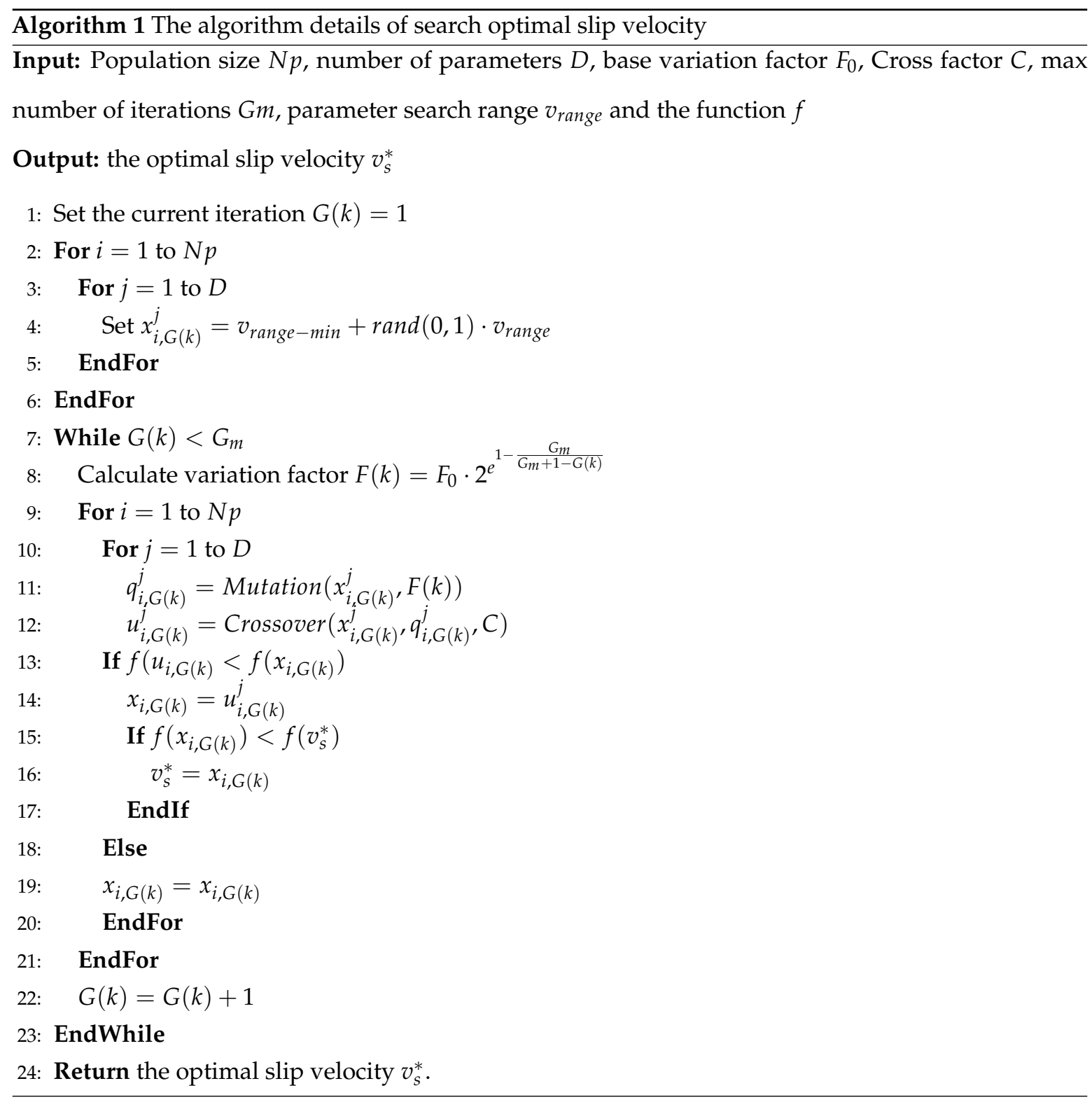

\section{Super-Twisting Sliding Mode Controller}

\subsection{Controller Design}

As a classical switching control, sliding mode control algorithms have been widely used in many fields [25]. Although the general first-order sliding mode controller has fast tracking speed and small steady-state error, it has a fatal disadvantage. The first-order sliding mode controller has more severe vibration. Consider the combination of constant velocity and exponential approach law in general first-order sliding mode control. Let slip surface

$$
\dot{s}=-\gamma_{1} s-\gamma_{2} \operatorname{sign}(s)
$$


where the $k_{1} s$ is exponential convergence term which ensures that the system can quickly converge near the sliding surface when away from the sliding surface. And the closer to the sliding surface, the lower the approach rate. The $k_{2} \operatorname{sign}(s)$ is isokinetic convergence term which can converge at a relatively uniform speed when the system moves near the origin. However, for any system, disturbances are unavoidable. Consider the following systems with disturbances

$$
\begin{gathered}
\dot{x}_{1}=x_{2} \\
\dot{x}_{2}=A+B u+\Delta A+\Delta B u+f_{0}
\end{gathered}
$$

where $\Delta A$ and $\Delta B$ respectively represent the disturbance of the system parameters, and $f_{0}$ represents unknown disturbances and unmodeled system states. Let $f=\Delta A+\Delta B+f_{0}$, and rewrite the system state equation as

$$
\begin{gathered}
\dot{x}_{1}=x_{2} \\
\dot{x}_{2}=A+B u+f
\end{gathered}
$$

If the above system is to be designed with a reaching law sliding mode controller, the specific value of perturbation $f$ cannot be obtained without using an observer. Design in accordance with the above sliding surface, and set the sliding surface $s=c e+e^{\prime}$. The system's sliding mode equation is

$$
\dot{s}=c \dot{x}_{1 r e f}-c x_{2}+\ddot{x}_{1 r e f}-A+B u=-\gamma_{1} s-\gamma_{2} \operatorname{sign}(s)+f
$$

Note that there is a symbolic function in the above formula, but most of the actual systems are discrete systems. In the case of a discrete system, the sliding mode dynamic equation of the system near the sliding mode surface is

$$
s_{k+1}=s_{k}-T_{s} \gamma_{2} \operatorname{sign}\left(s_{k}\right)
$$

The exponential approach term $\gamma_{1} s$ is negligible near the sliding surface due to its minimal effect. $T_{S}$ is the control period of the system. When moving near the sliding surface, the sliding modulus cannot be changed continuously. If $s$ is a positive minimum at a certain moment, there is

$$
s_{k+1}=0^{+}-T_{s} \gamma_{2} \operatorname{sign}\left(0^{+}\right)
$$

Conversely, when $s$ is a negative minimum at some point, there is

$$
s_{k+1}=0^{-}-T_{s} \gamma_{2} \operatorname{sign}\left(0^{-}\right)
$$

It can be seen from the above two formulas that the system cannot make a real sliding mode motion after reaching the sliding mode surface. The value of the sliding mode switching has been switched back and forth between $-T_{s} \gamma_{2}$ and $T_{s} \gamma_{2}$. This is the theory explanation of the chattering phenomenon. At the same time, when the control period of the system is longer and the gain item of symbolic function $\gamma_{2}$ is larger, the chattering of the system will become more obvious. However, in the presence of interference terms, in order to ensure the stability of the system, a sign function with a high gain term has to be introduced to suppress the disturbance, which will also exacerbate the chattering phenomenon.

If the above-mentioned shaking phenomenon occurs in the braking force control of a subway train, it is actually reflected that the passengers will obviously feel the front-to-back shake of the train body, which is caused by the large and small braking force. In addition, frequent chattering will bring additional energy consumption and wear to the actuator and accelerate its aging. Therefore, this paper introduces the super-twisting algorithm to suppress its chatter. The super-twisting algorithm is essentially a higher-order sliding mode control algorithm, which hides discontinuous symbolic functions into higher-order derivatives of sliding mode variables. Through the hidden processing, after several integration operations, the symbol function becomes a continuous function. At least 
it can be guaranteed that the first derivative of the sliding modulus does not contain discontinuous symbol function terms, and can be kept at 0 on the sliding mode surface which can liminate chattering in theory.

The control algorithm of the second-order sliding mode controller based on the super-twisting algorithm is as follows

$$
\begin{gathered}
\dot{s}=-k_{1}|s|^{\frac{1}{2}} \operatorname{sign}(s)+u_{s w} \\
\dot{u}_{s w}=-k_{2} \operatorname{sign}(s)
\end{gathered}
$$

where, $s$ is a sliding mode variable, $u_{s w}$ is an intermediate variable, and $k_{1}, k_{2}$ are parameters to be designed. Although there are two symbolic functions in the above mathematical expression of the controller, the first term will make $|s|^{\frac{1}{2}} \approx 0$. Symbolic function has almost no chattering effect on the system. The second symbolic function term is hidden in the derivative. In the first derivative of the sliding mode, the symbolic function after integration is transformed into a continuous function. Therefore, it can be guaranteed that the system has $s=s^{\prime}=0$ on the sliding surface.

For the problem of subway slip control, the goal of this article is to make the current slip speed of the subway train track the best slip speed estimated in the previous section. Thus, the tracking error is

$$
e=v_{s}-v_{\text {sref }}=v-\omega r-v_{\text {sref }}
$$

Because the linear velocity of the subway and the angular velocity of the wheels are not integral or differential, let sliding mode variables be $s=e$. The first derivative of the sliding surface is

$$
\dot{s}=\dot{v}-\dot{\omega} r-\dot{v}_{\text {sref }}
$$

Substitute into the subway kinematics model to obtain

$$
\dot{s}=-\frac{F_{x}+f+W}{m}-\frac{T_{b} r}{J}-\frac{F_{x} r^{2}}{J}-\dot{v}_{\text {sref }}
$$

Combining the above sliding mode variables based on the super-twisting algorithm, we get

$$
-\frac{F_{x}+f+W}{m}-\frac{T_{b} r}{J}-\frac{F_{x} r^{2}}{J}-\dot{v}_{\text {sref }}=-k_{1}|s|^{\frac{1}{2}} \operatorname{sign}(s)-k_{2} \operatorname{sign}(s)
$$

Since $T_{b}$ is the control variable of the system, the controller output is

$$
u=T_{b}=\frac{J}{r}\left[k_{1}|s|^{\frac{1}{2}} \operatorname{sign}(s)+k_{2} \int \operatorname{sign}(s)+\Phi-\dot{v}_{\text {sref }}\right]
$$

where

$$
\Phi=-\frac{F_{x}+f+W}{m}-\frac{F_{x} r^{2}}{J}
$$

Bringing it back to the (28) and (29), and ignoring the constant terms. There is

$$
\begin{gathered}
\dot{s}=-k_{1}|s|^{\frac{1}{2}} \operatorname{sign}(s)+u_{s w}+\frac{f+W}{m} \\
\dot{u}_{s w}=-k_{2} \operatorname{sign}(s)
\end{gathered}
$$

It can be seen that the disturbance term exists in the first derivative of the sliding modulus. The combination of the disturbance term and the intermediate variable can be obtained by differentiating it.

$$
\dot{s}=-k_{1}|s|^{\frac{1}{2}} \operatorname{sign}(s)+u_{s w}
$$




$$
\dot{u}_{s w}=-k_{2} \operatorname{sign}(s)+\frac{\dot{f}+\dot{W}}{m}
$$

As can be seen from the above formula, after the high-order sliding mode controller of the super-twisting algorithm, the first derivative of the sliding modulus does not include the disturbance term, so the controller is very robust to external disturbances. The stability of the controller is analyzed below.

\subsection{Controller Stability Proof}

According to Lyapunov's theorem, when the Lyapunov function of a system is positive definite and its derivative is negatively timed, the entire system is progressively stable. For this controller, take the state variable as

$$
X^{T}=\left[\begin{array}{c}
|s|^{\frac{1}{2}} \operatorname{sign}(s) \\
u_{s w}
\end{array}\right]=\left[\begin{array}{l}
x_{1} \\
x_{2}
\end{array}\right]
$$

Construct a Lyapunov function by the form

$$
V=\frac{1}{2} X^{T} Q X
$$

where $Q \in \mathbb{R}^{2 \times 2}$

$$
Q=\left[\begin{array}{cc}
4 k_{2}+k_{1}^{2} & -k_{1} \\
-k_{1} & 2
\end{array}\right]
$$

When $k_{2}>0, Q$ is positive definite matrix and $V$ is quadratic form of positive definite matrix. The air resistance and the curve additional resistance are bounded. Let there be a constant $\Delta$, so that the derivative of the perturbation can satisfy

$$
\left|\frac{\dot{f}+\dot{W}}{m}\right|<\Delta
$$

When the parameters $k_{1}, k_{2}$ satisfy the following conditions [26]

$$
\left\{\begin{array}{l}
k_{1}>2 \\
k_{2}>\frac{k_{1}^{3}+\Delta^{2}\left(4 k_{1}-8\right)}{k_{1}\left(4 k_{1}-8\right)}
\end{array}\right.
$$

The first derivative of the Lyapunov function can be made to negative definite, so the system is asymptotically stable. It means that the sliding mode variable and the derivative of the sliding mode variable in the sliding mode controller of the super-twisting algorithm can converge to the origin in a limited time, so that the system overcomes the disturbance and reaches the sliding mode.

\section{Simulation Analysis}

In this paper, the Simulink toolbox in MATLAB is used to simulate the estimation of the optimal online slip speed on dry, wet, and snow and ice surfaces, and the optimal slip based on the super-twisting sliding mode controller is used for speed tracking control. The simulation parameters are set as Table 3.

The parameters for traditional sliding mode controller are given as $\gamma_{1}=36, \gamma_{2}=12$. And the sliding manifold is given as $\dot{s}=-\gamma_{1} s-\gamma_{2} \operatorname{sign}(s)$. The parameters for proposed controller are given as $k_{1}=2000, k_{2}=1.5$.

In this simulation scenario, the subway train first runs on the dry rail surface, and keeps decelerating at the optimal slip speed, then drives into the wet rail surface area, and finally into the snow and ice rail surface area. 
Table 3. Parameters of Simulation.

\begin{tabular}{ccc}
\hline Parameters' Name & Symbol & Value \\
\hline Wheel load of subway & $m$ & $8000 \mathrm{~kg}$ \\
Wheel inertia & $J$ & $240 \mathrm{~kg} \cdot \mathrm{m}^{2}$ \\
Wheel radius & $r$ & $0.5 \mathrm{~m}$ \\
Length of subway & $l_{t}$ & $136 \mathrm{~m}$ \\
\hline
\end{tabular}

When driving on different rail surfaces, based on the recursive least squares and differential evolution methods used in this paper, the estimated adhesion state of the wheels and the actual theoretical adhesion characteristics are shown in the Figure 5.

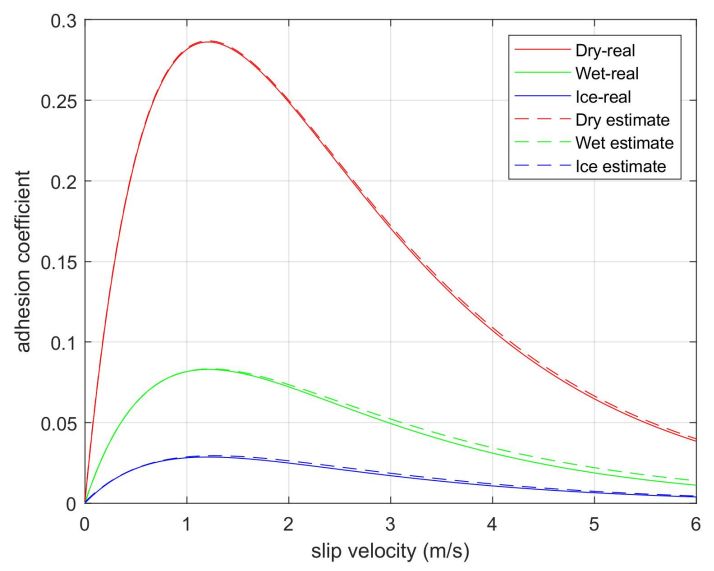

Figure 5. Theoretical adhesion characteristic curves under different wheel-rail contact conditions and the estimated curves of this method. The red curve is the adhesion characteristic curve of the dry rail surface. The green curve is the adhesion characteristic curve of the wet rail surface. The blue is the adhesion characteristic curve of the snow and ice rail surface. The solid line is the actual characteristic, and the dotted line is the estimated curve.

It can be seen from the above figure that the method used in this article estimates the adhesion area in the adhesion characteristic curve accurately, and the estimation has a relatively obvious error at high slip speeds, but this does not affect the real-time estimation of the subway operation because subway trains always work in the adhesion zone, and the goal controlled by this article is to make the slip speed of the subway at the peak of the adhesion zone but not exceed the adhesion zone and enter the slip zone. The estimation errors under different rail surface are as Table 4.

Table 4. The estimation errors under different rail surface.

\begin{tabular}{cc}
\hline Rail Surface Conditions & The Estimation Errors \\
\hline Dry & $8.0239 \times 10^{-5}$ \\
Wet & $2.0435 \times 10^{-6}$ \\
Ice & $5.1227 \times 10^{-6}$ \\
\hline
\end{tabular}

As can be seen from the Figure 6, the train is on a dry track at $t=0-2 \mathrm{~s}$. At $t=2 \mathrm{~s}$, the subway moves from the dry track to the wet track, the wheel slip speed increases, and The train has a tendency to move from the sticky zone to the slip zone. The method in this paper can estimate the optimal speed of the current track surface state within $0.5 \mathrm{~s}$, and maintain stability. The updated control target is the current optimal slip velocity. On the premise that the deceleration requirements are met as far as 
possible, the subway will not run in the slip zone. At $t=4 \mathrm{~s}$, the subway runs from the wet track to the snow track, and the slip speed increases again. The method in this paper can estimate the optimal slip speed at this time within $0.5 \mathrm{~s}$ and update it to the control to avoid entering the slip zone.

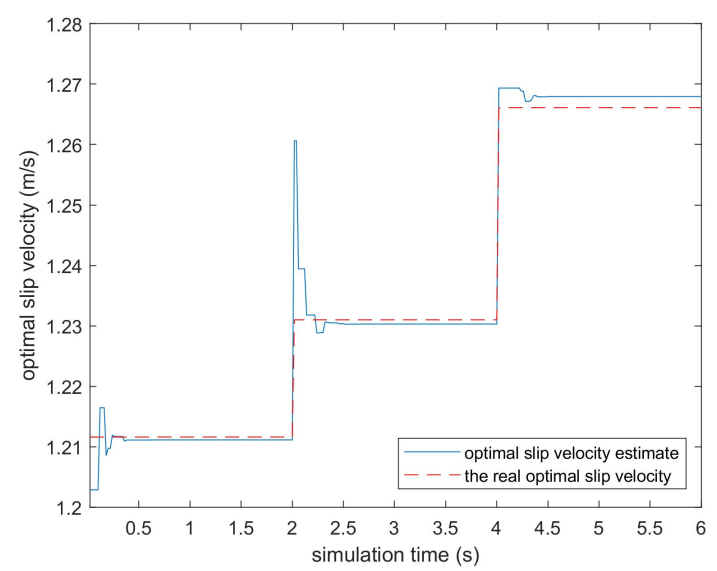

Figure 6. The optimal slip velocity is estimated under different rail conditions. The blue solid line is the curve estimated by the method in this paper, and the red dotted line is the actual optimal slip velocity.

After estimating the current optimal slip speed under different track surface conditions, the anti-slip controller will target the slip speed and control the braking torque so that the current subway train tracks the target slip speed while meeting the deceleration requirements as much as possible In order to maximize the use of wheel-rail adhesion. The slip speed tracking curve is shown in Figure 7. It can be seen that the super-twisting sliding mode controller used in this paper is faster than the traditional sliding mode controller, and is more stable in the steady state, that is, when the system is close to the sliding mode surface. Among them, a partial enlarged view is shown in the Figure $7 \mathrm{~b}-\mathrm{d}$.

It can be seen from the partial enlarged view Figure $7 \mathrm{~b}-\mathrm{d}$, that the traditional sliding mode controller will always tremble near the surface of the sliding mode. This is not a favorable phenomenon for the subway, and it will cause the brake actuator to change its output and accelerate the aging of the basic braking part. In addition, the braking torque is constantly changing, which will cause the deceleration to change continuously, causing minor vibrations in the entire subway car and significantly reducing passenger comfort.

As can be seen from the Figure 8, the output of the controller based on the traditional sliding mode control algorithm has been constantly jittering within a range, which also confirms the theoretical analysis in the previous section. In the first $2 \mathrm{~s}$, the subway is on a dry track surface, and the adhesion coefficient between wheels and rails is high, which can provide a relatively large braking force when braking. Therefore, it can be seen from the figure that the output of the braking torque is also relatively high. During 2 to $4 \mathrm{~s}$, when entering the wet track surface, the maximum adhesion force provided by the track surface does not support the subway to brake at the previous deceleration. Therefore, the controller needs to reduce the braking torque appropriately to avoid the wheels from violently slide. After $4 \mathrm{~s}$, the subway enters a smoother snow and ice track surface, the braking force provided by the track surface is smaller, so the braking force needs to be further reduced. However, although the braking torque of the subway has been decreasing as a whole, the braking torque has been trembling, which is very unfriendly to the braking components of the vehicle, and also reduces the comfort. 

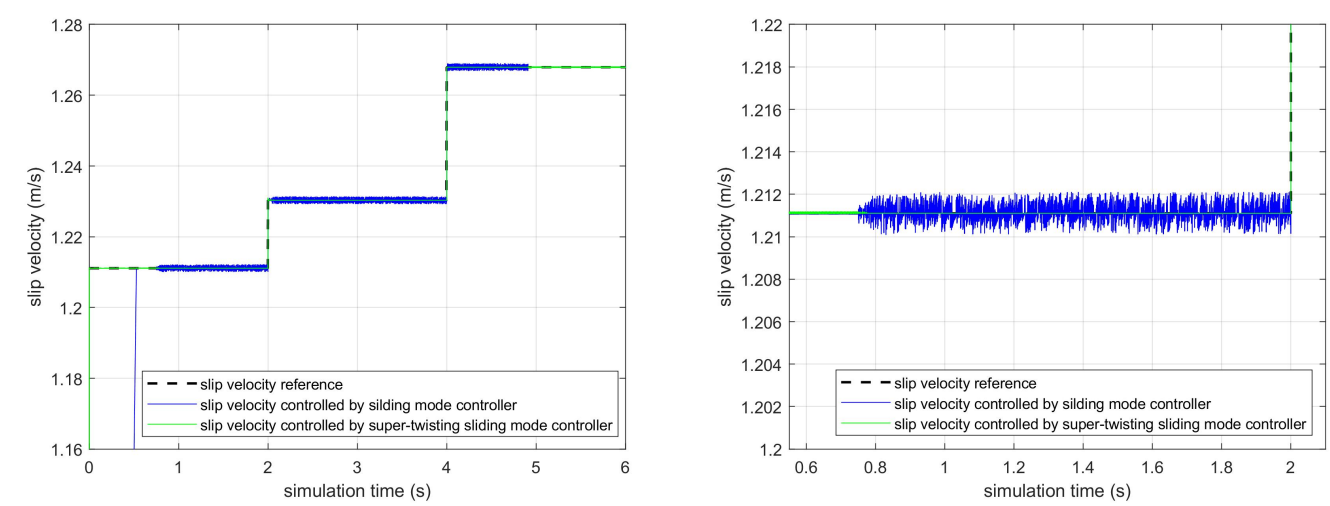

(a) The entire view of the best slip velocity tracking curve(b) Partially enlarged view of the best slip velocity tracking when the subway is running on the dry, wet and ice rail. curve when the subway is running on the dry rail.
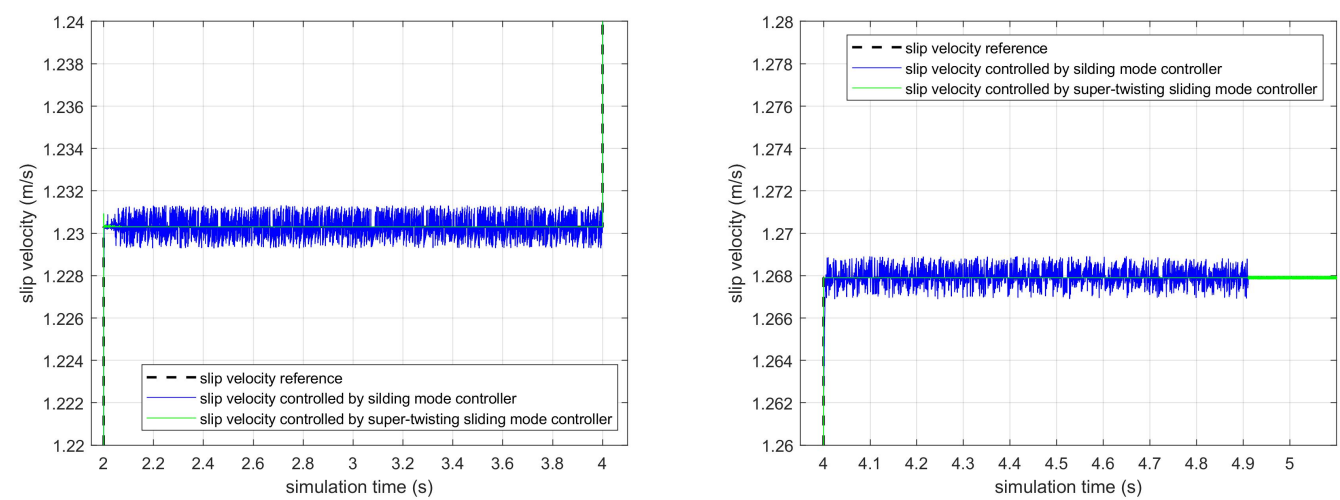

(c) Partially enlarged view of the best slip velocity tracking(d) Partially enlarged view of the best slip velocity tracking curve when the subway is running on the wet rail. curve when the subway is running on the ice rail.

Figure 7. Optimal slip velocity tracking curve controlled by sliding mode controller and super-twisting sliding mode controller. The black dotted line is the reference value of the optimal slip velocity estimated in the previous steps, the solid blue line is the tracking curve based on the sliding mode controller control, and the solid green line is the sliding velocity tracking based on the super-twisting sliding mode controller curve.

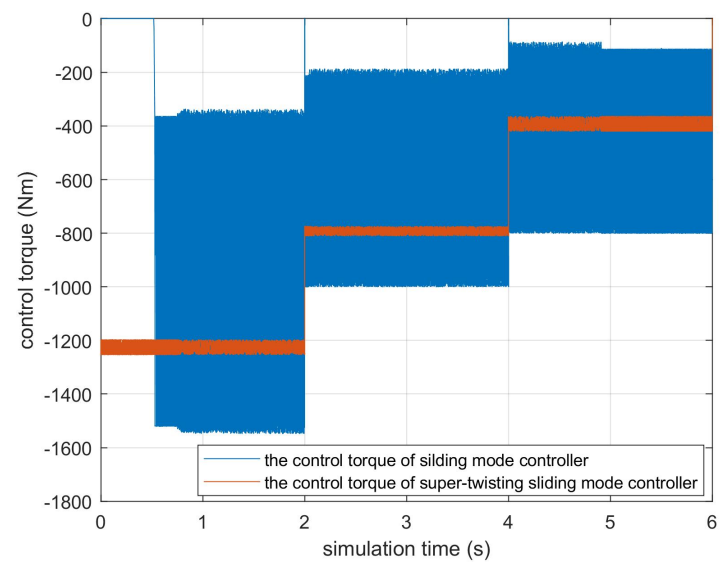

Figure 8. The control torque of sliding mode controller and super-twisting sliding mode controller proposed bu this paper. The blue curve is the control torque of sliding mode controller and the red curve is the control torque of super-twisting sliding mode controller. 
The output of the improved sliding mode controller based on super-twisting algorithm used in this article is shown in the Figure 8. It can be seen from the figure that the super-twisting sliding mode controller proposed by this paper can better suppress the chattering problem derived in the previous section. Makes the subway more stable and can achieve more accurate parking during braking.

The following Figure 9 shows the control error of the two control algorithms. The blue curve is the control error of the traditional sliding mode controller, and the red curve is the control error of the sliding mode controller based on the super-twisting algorithm in this paper. It can be seen from the figure that the control error of the method used in this paper is significantly smaller than that of the traditional sliding mode controller. There is a significant abrupt change in the error in the second and fourth seconds in the picture, which is due to the optimal slip velocity change caused by the subway vehicle running from the dry rail surface to the wet rail surface and from the wet rail surface to the snow and ice rail surface.

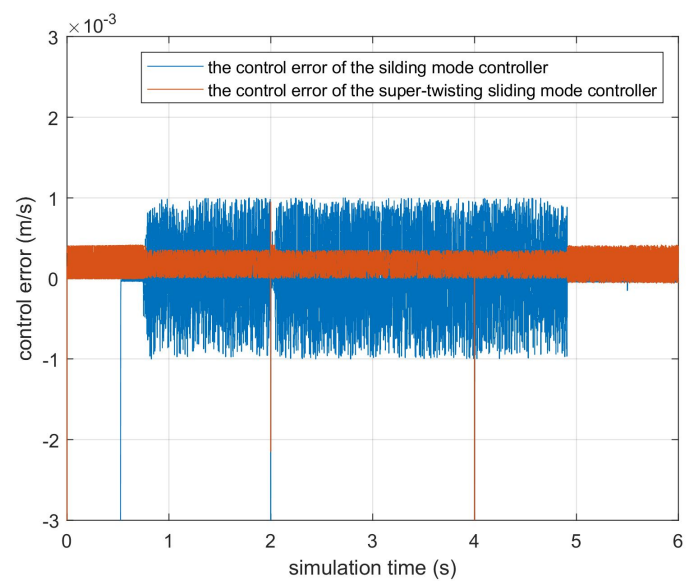

Figure 9. The error of sliding mode controller and super-twisting sliding mode controller proposed by this paper. The blue curve is the control error of the traditional sliding mode controller, and the red curve is the control error of the super-twisting sliding mode controller in this paper.

The simulation results show that the proposed algorithm is effective and superior when the metro train is running in a straight line. However, in the subway operation process, the subway will inevitably run on the curved track. Unlike a straight line, when the train travels on a curve, the air resistance and the additional resistance of the curve of the track needs to be considered. In the following simulation, the tracking effect of slip velocity of a subway train in the curve is considered, and the remaining parameters are consistent with the previous simulation. In this simulation, two curves are added. The subway train enters the first curve at the $t=2 \mathrm{~s}$, and the curve radius is $R_{1}=800 \mathrm{~m}$. At $t=4 \mathrm{~s}$, the subway train enters the second curve, and the curve radius is $R_{2}=400 \mathrm{~m}$. The Figure 10 shows the optimal slip velocity tracking curve with curves.

The red dashed line in the Figure 10 shows the tracking effect of slip velocity when the subway train enters the curve. From the tracking curve of slip velocity, it can be seen that after the subway train enters the curve, the time to reach the optimal slip velocity is longer than that of the straight line due to the action of the resistance of the curve accessory, which increases by about $15 \%$. However, the tracking of the target slip velocity is still guaranteed within $0.1 \mathrm{~s}$. Moreover, due to the effect of the super-twisting algorithm, the proposed method is better than the traditional sliding mode control algorithm in the curve. The response time of the proposed algorithm is shorter than that of the traditional sliding mode control algorithm. In particular, the smaller the curve radius, that is, the greater the degree of bending, the longer the adjustment time, but it can be controlled within the required response time range. Figure 11 shows the output curves of the control quantity of the two controllers in the presence of curves. 


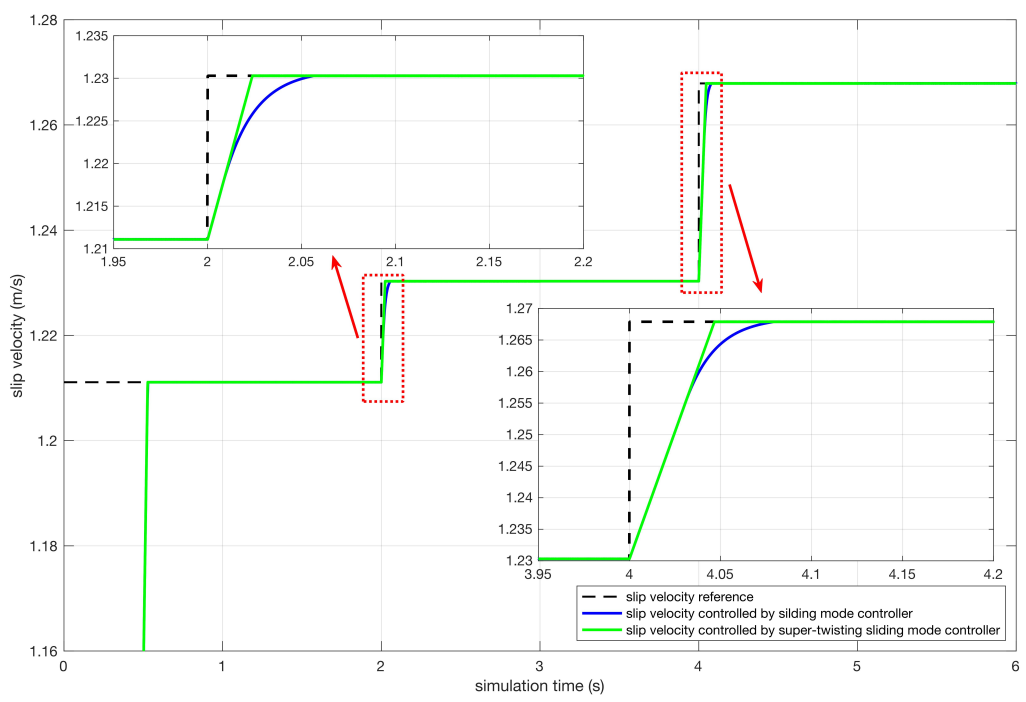

Figure 10. Optimal slip velocity tracking curve controlled by sliding mode controller and super-twisting sliding mode controller which includes curve rail.

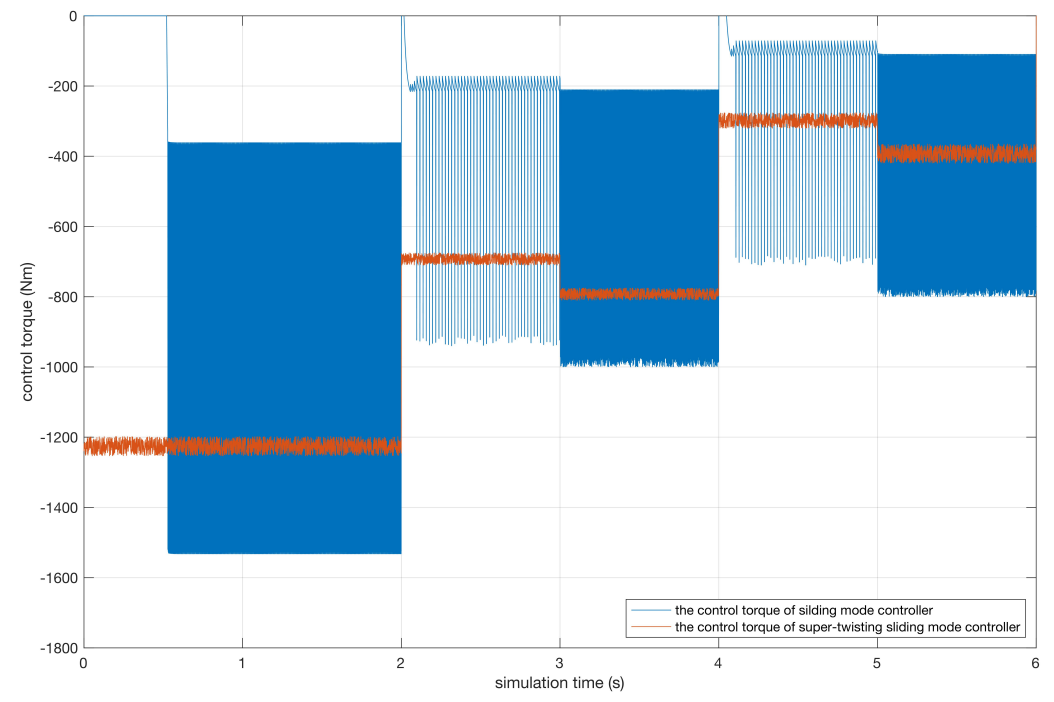

Figure 11. The control torque of sliding mode controller and super-twisting sliding mode controller proposed bu this paper includes curve rail.

As can be seen from Figure 11, the method presented in this paper can still achieve a relatively stable output without excessive shaking when there are curves. In addition, the output of control quantity is relatively stable in the adjustment process. However, the traditional sliding mode control method has a large amplitude of output variation and a large degree of shaking when the subway train enters the curve.

\section{Conclusions}

The purpose of this paper was to introduce an online super-twisting sliding mode anti-slip control strategy for subways. The recursive least squares was used to estimate the adhesion state of wheel-rail and the differential evolution algorithm was adopted to search for the optimal slip velocity in a short time. Besides, the super-twisting sliding mode controller was designed to eliminate chattering when 
the system was stable as well as reduce the control errors. Experimental results show that the proposed approach in this paper can estimate the wheel-rail adhesion state and search for optimal slip velocity in less than half a second in every control period. Besides, the chattering vibration was eliminated and the control error was reduced greatly compared with a slide mode controller.

Author Contributions: Conceptualization, Z.H. and W.D.; methodology, W.D. and B.C.; software, W.D.; validation, W.D.; formal analysis, X.T.; investigation, Y.L.; resources, B.C. and K.G.; data curation, Y.Y.; writing-original draft preparation, W.D.; writing-review and editing, Y.L. and X.T.; visualization, Y.Y.; supervision, Z.H.; project administration, Z.H. and K.G.; funding acquisition, Z.H. and W.D. All authors have read and approved the final version of the manuscript.

Funding: This research was funded by the National Natural Science Foundation of China (Grant Nos.61672537, 61672539 and 61873353).

Acknowledgments: This research was funded by the National Natural Science Foundation of China (Grant Nos. 61672537, 61672539 and 61873353).

Conflicts of Interest: The authors declare no conflict of interest.

\section{Nomenclature}

$\begin{array}{llll}r & \text { Wheel radius }(\mathrm{m}) & P_{k} & \text { Inverse correlation matrix at moment } k \\ v & \text { Vehicle body linear speed }(\mathrm{m} / \mathrm{s}) & g_{k} & \text { Gain vector at moment } k \\ T_{b} & \text { Braking torque }(\mathrm{N} \cdot \mathrm{m}) & \xi_{k} & \text { Prior estimation error } \\ \omega & \text { Wheel speed }(\mathrm{rad} / \mathrm{s}) & G(k) & \text { Current generation } \\ v_{s} & \text { Slip velocity }(\mathrm{m} / \mathrm{s}) & F(k) & \text { Variance factor at generation } G(k) \\ \eta & \text { Slip ratio } & G_{m} & \text { Max number of iteration } \\ f & \text { Air resistance }(\mathrm{N}) & N p & \text { Population size } \\ J & \text { Inherit of the wheel }\left(\mathrm{kg} \cdot \mathrm{m}^{2}\right) & \mathrm{D} & \text { Number of parameters } \\ m & \text { Wheel load }(\mathrm{kg}) & \mathrm{C} & \text { Cross factor } \\ F_{a} & \text { Adhesion force }(\mathrm{N}) & F_{0} & \text { Base variation factor } \\ \mu & \text { Adhesion coefficient } & s & \text { Sliding surface } \\ c_{1}, c_{2}, a, b & \text { Parameters of Burckhardt's model } & T_{s} & \text { Sample period }(\mathrm{s}) \\ \epsilon_{i} & \text { Estimate error } & k_{1}, k_{2} & \text { Parameters of controller } \\ \lambda & \text { Forgetting factor } & s i g n(\cdot) & \text { Symbolic function } \\ e & \text { Control error }(\mathrm{m} / \mathrm{s}) & \mu_{0}, \mu_{1}, \mu_{2} & \text { Constant value of air resistance } \\ R & \text { Radius of the curve }(\mathrm{m}) & W & \text { Curve additional resistance }(\mathrm{N}) \\ l_{t} & \text { Length of subway }(\mathrm{m}) & l_{r} & \text { Length of curve (m) }\end{array}$

\section{References}

1. Singh, M.; Singh, D.; Jara, A. Secure cloud networks for connected \& automated vehicles. In Proceedings of the IEEE International Conference on Connected Vehicles and Expo (ICCVE), Shenzhen, China, 19-23 October 2015; pp. 330-335.

2. Clerk Maxwell, J. A Treatise on Electricity and Magnetism; Clarendon: Oxford, UK, 1892; Volume 2.

3. Park, S.H.; Kim, J.S.; Choi, J.J.; Yamazaki, H.O. Modeling and control of adhesion force in railway rolling stocks. IEEE Control Syst. Mag. 2008, 28, 44-58.

4. Yin, D.; Sun, N.; Shan, D.; Hu, J.S. A multiple data fusion approach to wheel slip control for decentralized electric vehicles. Energies 2017, 10, 461. [CrossRef]

5. Yamazaki, H.o.; Nagai, M.; Kamada, T. A study of adhesion force model for wheel slip prevention control. JSME Int. J. Ser. C Mech. Syst. Mach. Elem. Manuf. 2004, 47, 496-501. [CrossRef]

6. Frylmark, D.; Johnsson, S. Automatic Slip Control for Railway Vehicles. Master's Thesis, Linkoping University, Linkoping, Sweeden, 2003.

7. Hsiao, T. Robust estimation and control of tire traction forces. IEEE Trans. Veh. Technol. 2012, 62, 1378-1383. [CrossRef] 
8. Nabizadeh, E.; Javadi, S.; Sabbaghi-Nadooshan, R. A fuzzy model for slip control in rail transportation systems. Asin Trans. Fundam. Electron. Commun. Multimed. 2011, 1, 7-12.

9. Park, S.H.; Kim, J.S.; Choi, J.J. Reference slip ratio generation and adaptive sliding mode control for railway rolling stocks. Int. J. Precis. Eng. Manuf. 2009, 10, 39-44. [CrossRef]

10. Precup, R.E.; Preitl, S.; Radac, M.B.; Petriu, E.M.; Dragos, C.A.; Tar, J.K. Experiment-based teaching in advanced control engineering. IEEE Trans. Educ. 2010, 54, 345-355. [CrossRef]

11. Sadr, S.; Khaburi, D.A.; Rodríguez, J. Predictive slip control for electrical trains. IEEE Trans. Ind. Electron. 2016, 63, 3446-3457. [CrossRef]

12. Rath, J.J.; Veluvolu, K.C.; Defoort, M.; Soh, Y.C. Higher-order sliding mode observer for estimation of tyre friction in ground vehicles. IET Control Theory Appl. 2014, 8, 399-408. [CrossRef]

13. Vo-Duy, T.; Ta, M.C. Slip Ratio Estimation for Traction Control of Electric Vehicles. In Proceedings of the IEEE Vehicle Power and Propulsion Conference (VPPC), Chicago, IL, USA, 27-30 August 2018; pp. 1-6.

14. He, J.; Liu, G.; Liu, J.; Zhang, C.; Cheng, X. Identification of a Nonlinear Wheel/Rail Adhesion Model for Heavy-Duty Locomotives. IEEE Access 2018, 6, 50424-50432. [CrossRef]

15. Pichlík, P.; Zděnek, J. Extended Kalman filter utilization for a railway traction vehicle slip control. In Proceedings of the IEEE International Conference on Optimization of Electrical and Electronic Equipment (OPTIM) \& 2017 Intl Aegean Conference on Electrical Machines and Power Electronics (ACEMP), Fundata, Romania, 25-27 May 2017; pp. 869-874.

16. Xu, X.; Peng, J.; Zhang, R.; Chen, B.; Zhou, F.; Yang, Y.; Gao, K.; Huang, Z. Adaptive Model Predictive Control for Cruise Control of High-Speed Trains with Time-Varying Parameters. J. Adv. Transp. 2019, 2019, doi:10.1155/2019/7261726. [CrossRef]

17. Strano, S.; Terzo, M. On the real-time estimation of the wheel-rail contact force by means of a new nonlinear estimator design model. Mech. Syst. Signal Process. 2018, 105, 391-403. [CrossRef]

18. Onat, A.; Voltr, P. Swarm intelligence based multiple model approach for friction estimation at wheel-rail interface. In Proceedings of the 5th International Symposium on Engineering, Artificial Intelligence and Applications (ISEAIA 2017), Kyrenia, Cyprus, 8-10 August 2017.

19. Uyulan, C.; Gokasan, M.; Bogosyan, S. Re-adhesion control strategy based on the optimal slip velocity seeking method. J. Mod. Transp. 2018, 26, 36-48. [CrossRef]

20. Gao, K.; Huang, S.; Han, F.; Li, S.; Wu, W.; Du, R. An Integrated Algorithm for Intersection Queue Length Estimation Based on IoT in a Mixed Traffic Scenario. Appl. Sci. 2020, 10, 2078. [CrossRef]

21. Baek, J.; Jin, M.; Han, S. A new adaptive sliding-mode control scheme for application to robot manipulators. IEEE Trans. Ind. Electron. 2016, 63, 3628-3637. [CrossRef]

22. Baek, S.; Baek, J.; Han, S. An adaptive sliding mode control with effective switching gain tuning near the sliding surface. IEEE Access 2019, 7, 15563-15572. [CrossRef]

23. Jin, X.; Zhang, W.; Zeng, J.; Zhou, Z.; Liu, Q.; Wen, Z. Adhesion experiment on a wheel/rail system and its numerical analysis. Proc. Inst. Mech. Eng. Part J J. Eng. Tribol. 2004, 218, 293-304. [CrossRef]

24. Ohyama, T. Some basic studies on the influence of surface contamination on adhesion force between wheel and rail at higher speeds. Railw. Tech. Res. Instit. Q. Rep. 1989, 30.

25. Li, H.; Zhang, X.; Peng, J.; He, J.; Huang, Z.; Wang, J. Cooperative CC-CV Charging of Supercapacitors Using Multi-Charger Systems. IEEE Trans. Ind. Electron. 2020, doi:10.1109/TIE.2019.2962485. [CrossRef]

26. Moreno, J.A.; Osorio, M. A Lyapunov approach to second-order sliding mode controllers and observers. In Proceedings of the 47th IEEE Conference on Decision and Control, Cancun, Mexico, 9-11 December 2008; pp. 2856-2861.

(C) 2020 by the authors. Licensee MDPI, Basel, Switzerland. This article is an open access article distributed under the terms and conditions of the Creative Commons Attribution (CC BY) license (http://creativecommons.org/licenses/by/4.0/). 